\title{
METHODS FOR PHYSICAL ACTIVITY EVALUATION AMONG THE WORKING POPULATION
}

\author{
Bislimovska Dragana \\ Institute of Occupational Health of R.N. Macedonia- Skopje, \\ WHO Collaborating Center, R.N. Macedonia, Faculty of Medicine, \\ Ss. Cyril and Methodius University in Skopje, R.N. Macedonia \\ e-mail: dbislimovska@gmail.com
}

\begin{abstract}
The high incidence of physical inactivity in the last decades has increased the need for development and use of lifestyle interventions that promote physical activity, especially among the working population.

The levels of occupational and leisure time physical activity differ and are specific among workers form different occupations, often creating opposing health effects or the so called "physical activity health paradox".

The aim of this paper was to present different methods for physical activity evaluation, and to determine the best methodological approach for the evaluation of the physical activity and its domains among the working population.

Literature review revealed that the most common methods for physical activity evaluation among the working population are: subjective (interview, self-reported questionnaires and physical activity logs), objective (measurement of energy expenditure, physiological measurements, use of motion sensors) and combined methods (subjective-objective).

Criteria for selection of corresponding methodological approach for physical activity evaluation among the working population are discussed, including the characteristics of the examined population and the used methods (quality, objectivity, level of workload, cost-effectiveness, specific limitations).

The obtained data will help creating recommendations for interventions in health promotion among the working population. methods

Keywords: physical activity health paradox, workers, subjective methods, objective

\section{Introduction}

Physical activity

Physical activity is defined as "any bodily movement produced by skeletal muscles that results in energy expenditure", and includes activities in the daily life that take place while the person is working, commuting in the household and during leisure time [1].

Physical activity can be classified as structured or unstructured activity, based on four basic dimensions: mode or type of activity, frequency, duration and intensity of the performed activity. In the assessment of physical activity and its effects, it is necessary to perceive the different domains of physical activity: occupational, transportation or utilitarian (e.g. walking to/ from a place, use of public transport, climbing/descending stairs), household and leisure time $[1,2]$.
\end{abstract}


In the evaluation of the health effects and gains of physical activity, it is essential to analyze the total activity that estimates all four domains, because increase of physical activity in one of the domains (e.g. in the workplace) can be compensated with the decrease of activity in one of the other three domains (e.g. leisure time).

\section{Working population}

According to the Labor Regulations Law of R. N. Macedonia from 2015, a worker is defined as an individual who is employed on the basis of an employment contract [3]. According to the International Standard Classification of Occupations - ISCO-08 of the International Labor Organization - ILO from 2012, the term occupation is defined as "a set of jobs which main tasks and duties, organizationally and technologically, are characterized by a high degree of similarity, and are meant to be performed by one person, who owns a set of abilities, knowledge and skills needed for the job" [4].

Various different classifications of occupations, depending on their characteristics, exist. Few decades ago, a classification made by Brown et al., divided occupations into 8 different categories, according to the level of energy expenditures and occupational physical load - muscular work grades - sedentary, sedentary-to-light, light, light-to-moderate, moderate, moderate-toheavy, heavy and very heavy [5]. According to the actual classification of physical load at work, workers are categorized into two groups: manual (blue collar) and non-manual (white collar) workers. Because of the specific characteristics of some occupations and workplaces, where manual and mental competences are required for the work tasks and duties (e.g. surgeon), more precise classifications are needed [6].

According to ISCO-08, in relation to the skills, tasks, duties and workloads required, occupations are classified into 4 different groups.

The first group consists of occupations that involve performance of simple, routine manual or physical tasks, and require a certain level of physical force and endurance (e.g. cleaners, gardeners, factory workers, warehouse clerks, etc.).

The second group includes workplaces where use of machinery, tools and electronic equipment is needed; precise manual work; knowledge of simple arithmetical calculations and good communication skills are vital (e.g. butchers, textile workers, professional drivers, hairdressers, police officers, etc.)

The third group involves occupations where performance of complex technical and practical duties and knowledge of specific technical procedures in a specialized field are essential (e.g. nurses, laboratory technicians, radiographers, legal secretaries, etc.).

The fourth group includes occupations in which the performance of the tasks and duties requires certain mental abilities to resolve complex problems, decision making and creativity (e.g. professors, musicians, doctors, engineers, etc.) [4].

\section{Health effects of physical activity among the working population}

In general, the health benefits from regular physical activity are:

- improved cardiorespiratory endurance,

- improved condition of the musculoskeletal system,

- reduced risk of hypertension, coronary heart disease, stroke, diabetes, different types of malignant diseases (e.g. breast cancer, colon cancer) and depression,

- reduced risk of trips and falls that can lead to fractures of the hip or spine,

- maintenance of healthy body weight and daily energy requirements [7].

According to the current recommendations for weekly physical activity from the World Health Organization (WHO), adult, active population, aged 18-64, should do at least 150 minutes of moderate-intensity or at least 75 minutes of vigorous-intensity aerobic physical 
activity or an equivalent combination of both. For additional health benefits, it is necessary to increase moderate-intensity aerobic physical activity to more than 300 minutes, combined with muscle-strengthening activities that involve all major muscle groups at least twice a week. These recommendations can be applied to physical activity in any of the four domains [8].

On the other hand, in certain occupations, specific differences in daily occupational and leisure time physical activity exist among the workers. High level of occupational physical activity is not always associated with positive health effects, but on the other hand, it is well documented workplaces with low level of physical activity - sedentary workplaces cause negative health effects [9].

The results from many research studies show that workers with physically demanding jobs have a higher risk of developing cardiovascular diseases than workers whose work does not involve physical effort. It is believed that this is due to the level of workload that workers are exposed to, as well as to the risk that increases with age [10]. Other studies have demonstrated the opposed health effects of occupational and leisure time physical activity, establishing the so-called physical activity health paradox [11].

The explanation behind the opposed health effects of occupational and leisure time physical activity arises from the differences in the physiological mechanisms they cause. Leisure time physical activity involves activities which type, duration and intensity can be individually determined. Usually, it consists of dynamic, aerobic activities that are short in duration and with sufficient rest between activities. On the other hand, occupational physical activity mainly incorporates static activities with long duration, limited time for recuperation, often monotonous and can't be fully controlled by the worker, straining the cardiovascular system $[12,13]$.

Finding a proper balance between occupational and leisure time physical activity is essential in preventing bad lifestyle among the working population. For people with sedentary work, leisure time physical activity is an efficient way of reducing negative health effects caused by sitting for long periods. On the other hand, for those working physically demanding jobs, although leisure time physical activity has positive health impact, it is very difficult to achieve daily recommendations, because of the amount of energy expenditure during working hours, causing lower motivation among the workers in leisure time activities [14].

\section{Aim}

The aim of this paper was to present different methods for physical activity evaluation, and to determine the best methodological approach for the evaluation of the physical activity and its domains among the working population. The obtained results will provide relevant recommendations for improving the health and workability of workers from different occupations.

\section{Methodology}

This paper represents a literature review. A computerized search for peer-reviewed original research and systematic literature reviews published in English after January 1, 2010 was used. PubMed and Web of Science databases were retrieved using the keywords: "physical activity" AND "methods for evaluation" AND "objective methods" OR "subjective methods".

\section{Physical activity evaluation methods}

Due to the high incidence of physical inactivity in the recent decades, there has been an increase in the development and application of various lifestyle interventions that promote physical activity in leisure time and encourage the working population to achieve public health recommendations for daily physical activity [15-17]. These interventions take into account individual, cultural, and environmental factors that influence health behavior and allow participants to individualize their physical activity programs according to their personal 
lifestyle $[15,16]$. The ability to adequately and effectively measure the impact of these interventions is crucial in the process of physical activity promotion $[15,18]$.

Physical activity and its domains are assessed by subjective and objective methods.

Subjective methods are based on the current recording of physical activity or recall of physical activity over a period of time, while objective methods are performed using devices carried by the participant that directly measure one or more biosignals, such as the acceleration of the body, heart rate or some other indicators of physical activity or energy expenditure.

There is no method that is considered the gold standard for assessing physical activity as it is a multifaceted and complex process, and the best results are obtained by combining two or more methods. Objective methods are more accurate than subjective ones, but, on the other hand, their application is usually limited to a smaller number of participants, as well as a shorter period of time monitoring their physical activity.

When choosing the most appropriate method for physical activity assessment for research purposes, it is necessary to take into account four basic elements: the quality of the measured physical activity (e.g. type of activity, intensity, frequency, duration), objectivity of the data, research participants burden (e.g. time and/or effort to perform the test), the cost-totime ratio required to perform the assessment and the specific limitations of each of the methods. It is also necessary to take into account the characteristics of the study population (gender, age, physical parameters, workplace, associated diseases, etc.) that may influence the choice of method or methods for evaluating physical activity [19].

The choice of method or methods also depends on the type of epidemiological study being conducted, the number of participants, the environment in which it is conducted, the costs associated with the study and the behavior of the respondents during the study. Certain methods, e.g. wearing activity monitors or the direct observation method can lead to the phenomenon of "reactivity", where the respondents begin to change their usual behavior and habits in relation to physical activity. On the other hand, methods based on self-reporting often lead to over-reporting of activities [20].

\section{Subjective methods}

There are two types of subjective methods that are used in the evaluation of physical activity: physical activity questionnaires and physical activity logs/diaries.

\section{Physical activity questionnaires}

Physical activity questionnaires are used to determine the types and dimensions of daily physical activity through interviewer-led or self-reported questionnaires. A number of questionnaires that vary in size exist, from short questionnaires that consist of few questions to long, detailed questionnaires that evaluate physical activity over certain period of time $[21,22]$.

The questionnaires also differ in the dimensions and domains of physical activity they evaluate (e.g. type, duration, frequency of activity), the method of assessment (e.g. duration of activity, energy expenditure in calories), quality of data (e.g. whether they can determine the difference between occupational and leisure time activity or between regular and new activities) and the type of data collection (e.g. computer based, interview, hard copy, etc.).

The main advantages of the use of questionnaires for physical activity evaluation are: the high cost-effectiveness of the method, due to the large number of participants that can be involved, the simple way of collection of data, the possibility for determining different types and intensity of activities (light, moderate and heavy/vigorous activity) for different time frames, the ability to group participants according to their level of physical activity and to follow the changes in daily activity over time, etc. On the other hand, the use of these questionnaires has certain limitations: the inability to differ certain dimensions of physical activity and their intensity (e.g. difference between light and moderate activity), the possibility of, 
intentionally or unintentionally, falsely answering some questions, etc. Limitations can occur if participants are unable to fully understand the language that is used in the questionnaires or some other factors, like the complexity of the questions, the age of the participants, the hours when the survey is conducted, etc. The questionnaires are more credible on a group than on individual level and when they are structured in chronological order for a certain period of time [18, 23].

Physical activity questionnaires are divided into three groups: global physical activity questionnaires, short recall physical activity questionnaires and quantitative history questionnaires.

The global questionnaires provide quick evaluation of the level of physical activity of the participants. Usually, these questionnaires are short (2-4 questions) and are used to determine whether respondents meet the recommended standards for daily physical activity, sorting them into two groups - active and inactive. These types of questionnaires are self-administered. The most commonly used global questionnaire is Exercise Vital Sign [24].

Short recall physical activity questionnaires are the most widely used type of questionnaires for physical activity evaluation in scientific research purposes as they enable fast and simple assessment of physical activity in all its dimensions and domains. These questionnaires usually consist of 7-12 questions that can be self-administered or can be used as an interview. They can determine the type of activity, its domain and the time spent in a particular type of activity during a month, week or day; they can also calculate scores for the intensity. The most commonly used questionnaires in this group are the International Physical Activity Questionnaire (IPAQ) and the Global Physical Activity Questionnaire (GPAQ) [23, 25]. These type of questionnaires have been applied in numerous surveys of the general and/or working population. Thus, Bennie et al. in a survey published in 2015, used the IPAQ questionnaire to assess sedentary behavior at leisure time and at work in administrative workers, while Medina et al. in 2013, and Moreira-Silva et al., also in 2013, for the assessment of physical activity in manual workers [26-28].

The GPAQ questionnaire, for example, was used to monitor physical activity in the office. It was used to evaluate workers in the chemical industry in the study by Wilke et al., from 2015, and in the study of Hanna et al., to assess sedentary behavior, physical activity and occurrence of back pain in Qatari university professors [29, 30]. In these studies, the previously mentioned advantages and limitations from the application of this type of questionnaires in the researches of the physical activity in different types of workers have been registered.

Quantitative questionnaires, on the other hand, are detailed surveys that are conducted over a long period of time (months or years), contain 20 to 60 questions, and are conducted in the form of an interview. They are used in epidemiological studies that evaluate the different types and intensity of physical activity from the past, that affect the morbidity and mortality from certain diseases and the current health status of the respondents. An example of this type of questionnaire is the Bone Loading History Questionnaire which assesses the impact of physical activity in childhood on the spine and hips in adulthood [23, 31].

\section{Physical activity logs/diaries}

The diaries/logs are used to obtain a detailed record of the respondent's physical activity. They can be used to assess the reliability and validity of the answers to physical activity questionnaires, and are usually used as an additional element in performing objective methods for assessing physical activity. The diaries are filled in by the respondents themselves, and contain data on the type of activity, the duration of the activity (the hour when it started and ended) and its intensity [31]. 


\section{Objective methods}

Objective methods for assessing physical activity are classified into: methods for measuring energy expenditure, physiological measurements, use of motion sensors and combined methods. As previously stated, the biggest limitation for the application of objective methods, for assessing physical activity for scientific research purposes, is the inability to include a large number of respondents given the unfavorable cost-benefit ratio due to the relatively complicated protocol for their performance, the need for adequately trained professional and technical staff, duration of the method, etc. The most commonly used objective method for assessing physical activity in the working population is the use of motion sensors [31].

\section{Methods for measuring energy expenditure}

This group of objective methods for assessing physical activity includes: indirect calorimetry, the doubly-labeled water method, and direct observation.

\section{Indirect calorimetry}

The assessment of energy consumption expenditure with indirect calorimetry is performed by measuring the ventilatory volume and determining the level of oxygen consumption in controlled conditions.

\section{Doubly-labeled water method}

This method determines the total energy expenditure in a period of one to three weeks, and if combined with the data from the measured energy expenditure at rest and the thermic effect of food, the doubly-labeled water method can calculate energy expenditure during physical activity.

The method is based on determining the difference in the elimination rate of two stable isotopes, oxygen (18O) and deuterium $(2 \mathrm{H})$. Known concentrations of these isotopes enter the body through water. Isotopes are distributed in body fluids, with deuterium eliminated from the body as water and oxygen isotopes in the form of water and carbon dioxide. The difference in the rate of elimination of these isotopes is represented by the production of carbon dioxide during the measurement through which the energy expenditure is then estimated [23,31].

\section{Direct observation}

During direct observation, an independent observer monitors and records the physical activity of a respondent. It is commonly used to assess physical activity in a confined space (e.g. for workers in a factory plant). The method provides data on the activity (type of activity, personal variations in performing the activity, etc.), as well as the time of day they perform a particular activity, favorite location, with whom the activity is performed, etc. [19, 23, 31].

\section{Physiological measurements}

\section{Heart rate monitoring}

Examination of physical activity with this method is performed by wearing a device that is placed around the wrist, and measures the heart rate by receiving signals from electrodes that are positioned on the chest of the test subject for a period of several days.

The measurement of heart rate in the assessment of physical activity is based on the physiological mechanism of changes in heart rate during any movement of the body, including physical activity, because the heart rate increases linearly and in proportion to the intensity of activity. The disadvantage of this method arises from the fact that the cardiac response to physical activity does not coincide instantly with energy expenditure, especially at the beginning and end of the activity, where the device either does not register the activity at all or overestimates the 
time spent in activities with higher intensity. The accuracy of the method is improved in such a way that, in addition to monitoring the heart rate, the oxygen consumption is also measured, which can determine the energy expenditure during a given activity [23, 32].

\section{Motion sensors}

Motion sensors are wearable devices that measure the movements of the human body, which can assess the energy expenditure during a particular physical activity. The most commonly used sensors in the assessment of physical activity are accelerometers and pedometers.

\section{Accelerometers}

Accelerometers are portable devices that can determine the frequency, duration and intensity of physical activity at certain time intervals by measuring the acceleration of the body during various movements.

Acceleration can be measured in one (usually vertical), two (vertical and medio-lateral or vertical and antero-posterior) or three planes (vertical, medio-lateral and antero-posterior). The device can be placed on the body on one or more locations (e.g. hip, upper or lower back, upper arm, etc.) on which the quality and accuracy of the obtained data will depend. Technological advances in recent decades have made accelerometers easy to carry due to their small size and the ability to record and store large amounts of data over a period of weeks.

The basic data obtained from the accelerometer are the acceleration and deceleration records of the body. These records are called raw accelerometer data and are recorded in gravitational acceleration units $(\mathrm{g})$, and are expressed as acceleration in meters per square second. Then, through sophisticated algorithmic software programs, this data are converted into measurable units through which the characteristics of physical activity are assessed. These can be presented in units that measure energy expenditure (e.g. kilocalories or MET), intensity of an activity (e.g. llight, moderate, high) or type of activity (e.g. standing, sitting) per units of time (minutes, hours etc.) [23, 32].

The application of accelerometers for scientific research purposes to assess the physical activity of the working population has become widespread in the last two decades. Thus, accelerometry as an objective method for assessing physical activity has been applied in the research of Skotte et al., from 2014, in which the various domains of physical activity in office workers were evaluated, in the research of Gupta et al., from 2016, which assessed the effect of physical activity from different domains on the occurrence of obesity in a group of Danish manual workers, and in a study by Stemland et al., from 2015, in which physical activity from different domains was evaluated in aircraft cabin crew [33-35].

The advantages of accelerometers are: the ability to continuously monitor the activity (from minute to minute), the accuracy in monitoring static and dynamic activities and the large memory capacity. Accelerometry, on the other hand, is an expensive method, especially if it analyzes a large group of subjects; it requires adequately trained professional and technical staff, as well as specialized hardware and software equipment. Additionally, there is no standard protocol for analyzing accelerometer data for research purposes. The use of these methods may also lead to reactive bias, i.e. the participant's behavior may change as a result of participating in the experiment [19].

\section{Pedometers}

Pedometers are devices that are used to assess physical activity through a sensor that counts the number of steps while walking. The number of steps is measured by analyzing the movements of the subject's hip which, when moving, accelerates in a vertical plane with a force greater than a certain selected threshold. Their simplicity, relatively low cost, and ability to recognize short periods of physical activity, that are often missed by questionnaires, make 
these devices suitable for use in assessing physical activity. Pedometers most accurately measure running and moderately brisk walking because these activities mostly involve moving forward in a vertical plane. The disadvantage of pedometers is the inability to register physical activity in a horizontal plane that occurs during periods of inactivity, leisure time or activities that only involve movement of the upper body.

There are a number of commercially available pedometer models, from simple ones that determine the number of steps and distance traveled to newer models with a built-in clock and the ability to determine time spent in activities of varying intensity. Depending on the manufacturer, pedometers differ in their vertical acceleration threshold, which leads to their distinct sensitivity and mechanism in obtaining results. Pedometers do not record the intensity of physical activity; they are not very accurate in tracking its frequency and duration and have a lower memory capacity than accelerometers. Due to these disadvantages, the results of the assessment of physical activity with this method are considered less relevant to the results obtained from accelerometers [19, 23, 32].

Pedometry has been used in many studies of physical activity in the working population in the last decade. Thus, in the research of Miller et al., pedometers were used as an objective method for assessing physical activity in different groups of workers in Australia; in Al-Mohannadi et al. research to assess Qatari health professionals and in Matthew et al. study, software engineers in India [36-38].

\section{Conclusion}

Several methods for assessing physical activity exist, but none of them is considered the gold standard. On the other hand, the physical activity of workers has a great impact on their health condition and work ability. Its assessment helps create recommendations for interventions for improvement of their health status. The most relevant results in assessing physical activity of the working population are expected to be obtained from a combination of subjective and objective methods, usually short recall physical activity questionnaires and the use of motion sensors, and their choice depends on the type, objectives and the duration of the research, as well as the characteristics of the participants involved.

Conflict of interest statement. None declared.

\section{References}

1. Caspersen CJ, Powell KE, Christenson GM. Physical activity, exercise, and physical fitness: definitions and distinctions for health-related research. Public Health Rep 1985; 100: 126-31.

2. Virkkunen H, Härmä M, Kauppinen T. The triad of shift work, occupational noise, and physical workload and risk of coronary heart disease. Occup Environ Med 2006; 63: 378-86.

3. Labor Regulations Law. Official Gazette of RM No. 165/2015. Available from: https://www.mtsp.gov.mk/content/pdf/zakoni/30.9_ZRO\%20precisten\%20167\%2015. pdf (Accessed 17.10.2021).

4. International Standard Classification of Occupations: ISCO-08. Geneva: International Labour Office - ILO; 2012.

5. Brown JR, Crowden GP. Energy expenditure ranges and muscular work grades. Brit J Industr Med 1963; 20: 277.

6. TeAra Encyclopedia of New Zealand. Defining and measuring occupations- Occupational structure. Ministry for Culture and Heritage TeManatuTaonga; 2016. Available from: https://teara.govt.nz/ (Accessed 24.10.2021). 
7. Amidu N, Owiredu WK, Mireku EK, Agyemang C. Metabolic syndrome among garage workers in the automobile industry in Kumasi, Ghana. J Med Biomed Sci 2012; 1: 29-36.

8. World Health Organization. Physical activity; 2018. Available from: https://www. who.int/news-room/fact-sheets/detail/physical-activity. (Accessed 17.10.2021).

9. Nooijen CFJ, del Pozo-Cruz B, Nyberg G, et al. Are changes in occupational physical activity level compensated by changes in exercise behavior?. Euro J Pub Health 2018; 28(5): 940-43.

10. Virkkunen H, Härmä M, Kauppinen T. The triad of shift work, occupational noise, and physical workload and risk of coronary heart disease. Occup Environ Med 2006; 63: 378-86.

11. Sorić M. Ključni pojmovi i epidemiologija tjelesne (ne)aktivnosti. In: Babić Z, Pintarić H, Mišigoj-Duraković M, Miličić D, editors. Sportska kardiologija - Kardiologija sporta, tjelesne i radne akivnosti. Zagreb: Medicinska naklada 2018; p: 14-20.

12. Clays E, Lidegaard M, De Bacquer D, et al. The combined relationship of occupational and leisure-time physical activity with all-cause mortality among men, accounting for physical fitness. Am J Epidemiol 2015; 179(559): 566.

13. Leino-Arjas P, Solovieva S, Riihimäki H, Kirjonen J, Telama R. Leisure time physical activity and strenuousness of work as predictors of physical functioning: a 28 year follow up of a cohort of industrial employees. Occup Environ Med 2004; 61(12): 1032-8.

14. Kirk MA, Rhodes RE. Occupation correlates of adults' participation in leisuretimephysical activity: a systematic review. Am J Prev Med 2011; 40: 476-85.

15. Silfee VJ, Haughton CF, Jake-Schoffman DE, et al. Objective measurement of physical activity outcomes in lifestyle interventions among adults: A systematic review. Prev Med Rep 2018; 11: 74-80.

16. Dunn AL, Andersen RE, Jakicic JM. Lifestyle physical activity interventions: history, short-and long-term effects, and recommendations. Am J Prev Med 1998; 15(4): 398-412.

17. Kahn EB, Ramsey LT, Brownson RC. The effectiveness of interventions to increase physical activity: a systematic review. Am J Prev Med 2002; 22(4): 73-107.

18. Kelly P, Fitzsimons C, Baker G. Should we reframe how we think about physical activity and sedentary behaviour measurement? Validity and reliability reconsidered. Int J Behav. Nutr Phys Act 2016; 13(1): 1.

19. Sylvia LG, Bernstein EE, Hubbard JL, Keating L, Anderson EJ. Practical guide to measuring physical activity. J Acad Nutr Diet. 2014;114(2):199-208.

20. Dowd KP, Szeklicki R, Minetto MA, Murphy MH, Polito A, Ghigo E, et al. A systematic literature review of reviews on techniques for physical activity measurement in adults: a DEDIPAC study. Int J Behav Nutr Phys Act 2018; 15(1): 1-33.

21. Jacobs DR, Ainsworth BE, Hartman TJ, Leon AS. A simultaneous evaluation of 10 commonly used physical activity questionnaires. Med Sci Sports Exerc 1993; 25: 81-91.

22. Strath SJ, Bassett DR, Swartz AM. Comparison of the College Alumnus Questionnaire physical activity index with objective monitoring. Ann Epidemiol 2004; 14: 409-15.

23. Strath SJ, Kaminsky LA, Ainsworth BE, Ekelund U, Freedson PS, Gary RA, Richardson CR, Smith DT, Swartz AM. Guide to the assessment of physical activity: clinical and research applications: a scientific statement from the American Heart Association. Circulation 2013; 128(20): 2259-79.

24. Quiles NN, McCullough AK, Piao L. Validity and Reliability of the Exercise Vital Sign Questionnaire in an Ethnically Diverse Group: A Pilot Study. J Prim Care Community Health 2019; 10: 2150132719844062.

25. Guidelines for Data Processing and Analysis of the International Physical Activity Questionnaire [IPAQ]-Short and Long Form, 2005. Available from: www.biobank. ctsu.ox.ac.uk (Accessed 15.09.2021). 
26. Bennie JA, Pedisic Z, Timperio A, Crawford D, Dunstan D, Bauman A, et al. Total and domain-specific sitting time among employees in desk-based work settings in Australia. Aust N Z J Public Health 2015; 39(3): 237-42.

27. Medina C, Barquera S, Janssen I. Validity and reliability of the International Physical Activity Questionnaire among adults in Mexico. Rev Panam Salud Publica 2013; 34(1): 21-8.

28. Moreira-Silva I, Azevedo J, Rodrigues S, Seixas A, Jorge M. Predicting musculoskeletal symptoms in workers of a manufacturing company. Int J Occup Saf Ergon 2020; 24: 1-9.

29. Wilke C, Ashton P, Elis T, Biallas B, Froböse I. Analysis of work ability and workrelated physical activity of employees in a medium-sized business. BMC Res Notes 2015; 8: 803.

30. Hanna F, Daas RN, El-Shareif TJ, Al-Marridi HH, Al-Rojoub ZM, Adegboye OA. The Relationship Between Sedentary Behavior, Back Pain, and Psychosocial Correlates Among University Employees. Front Public Health 2019; 7: 80.

31. Westerterp KR. Assessment of physical activity: a critical appraisal. Eur J Appl Physiol 2009; 105(6): 823-8.

32. Human Kinetics. Available methods for measuring physical activity; 2021. Available from: https://us.humankinetics.com/blogs/excerpt/available-methods-for-measuring-physicalactivity (Accessed 21.10.2021).

33. Skotte J, Korshøj M, Kristiansen J, Hanisch C, Holtermann A. Detection of physical activity types using triaxial accelerometers. J Phys Act Health 2014; 11(1): 76-84.

34. Gupta N, Hallman DM, Mathiassen SE, Aadahl M, Jørgensen MB, Holtermann A. Are temporal patterns of sitting associated with obesity among blue-collar workers? A cross sectional study using accelerometers. BMC Public Health 2016; 16: 148.

35. Stemland I, Ingebrigtsen J, Christiansen CS, Jensen BR, Hanisch C, Skotte J, Holtermann A. Validity of the Acti4 method for detection of physical activity types in free-living settings: comparison with video analysis. Ergonomics 2015; 58(6): 953-65.

36. Miller R, Brown W. Meeting physical activity guidelines and average daily steps in a working population. J Phys Act Health 2004; 1(3): 218-26.

37. Al-Mohannadi AS, Sayegh S, Ibrahim I, Salman A, Farooq A. Effect of a pedometerbased walking challenge on increasing physical activity levels amongst hospital workers. Arch Public Health 2019; 77: 40.

38. Mathew V, Akkilagunta S, Kumar D, Lakshminarayanan S, Kar SS. Effectiveness of Pedometer-Based Walking Program to Improve Physical Activity of Workers in a Software Industry: An Experimental Study. Int J Prev Med 2019; 10: 49. 